\title{
A multidimensional scaling analysis of vowel discrimination in humans and monkeys
}

\author{
JOAN M. SINNOTT, CHARLES H. BROWN, WASEEM T. MALIK, and REGINA A. KRESSLEY \\ University of South Alabama, Mobile, Alabama
}

\begin{abstract}
Multidimensional scaling (MDS) was used to compare perceptual maps for 10 synthetic English vowels in humans and Old World monkeys (Macaca fuscata and Cercopithecus albogularis). Subjects discriminated among the vowels using a repeating background procedure, and reaction times were submitted to an MDS analysis to derive measures of perceived similarity. The dimensions that emerged related to the frequencies of the first $(F 1)$, second $(F 2)$, and third $(F 3)$ formants. Human data indicated a good match to previous MDS studies using rating procedures or confusion matrices: The dominant dimension mapped onto vowel $F 2$, the phonetically most important formant, and the second and third dimensions mapped onto $F 1$ and $F 3$, respectively. For monkeys, equal weightings occurred for $F 1$ and $F 2$, and $F 3$ was not clearly represented. Monkey sensitivity to the formants appeared to relate to formant amplitudes. If monkeys are giving an accurate representation of the psychoacoustic relations among the formants, then our human results suggest that species-specific mechanisms, reflecting the salience of the phonetic feature of advancement, may contribute to vowel coding in humans.
\end{abstract}

Multidimensional scaling (MDS) has been in use for over 25 years to study the psychological space of human vowel systems (see, e.g., Fox, Flege, \& Munro, 1995; Pols, van der Kamp, \& Plomp, 1969; Rakerd, 1984; Rakerd \& Verbrugge, 1985; Shepard, 1972; Singh \& Woods, 1970). MDS is a mathematical tool that enables us to represent the similarities of perceptual objects spatially, as in a map (Schiffman, Reynolds, \& Young, 1981). For computation, we need a set of numbers that expresses all combinations of pairs of similarities within a group of objects. MDS procedures represent objects judged as similar to one another as points close to each other; dissimilar objects are represented as points distant from one another. MDS vowel data are typically derived from direct ratings of vowel similarity, or from confusion matrices obtained under nonoptimal listening conditions. In English, the vowel pairs $/ \mathrm{i} /-/ \mathbf{I} /, / \varepsilon /-/ \mathfrak{e} /, / \mathrm{a} /-/^{\wedge} /$, and $/ \mathrm{U} /-\mathrm{u} /$ tend to be confused and/or rated as highly similar. In MDS, such vowels materialize as points closer together in psychological space than the more dissimilar vowels.

Sinnott (1989) used a reaction time (RT) procedure to assess the spectral similarity of synthesized vowels. The advantage of this procedure was that it could easily be used with nonhuman primates in the context of a simple discrimination procedure to obtain comparative data. $\mathrm{Hu}-$

This work was supported by NIDCD Grants K04 DC 00042 and R01 DC 00541 to J.M.S. and R01 DC 00164 to C.H.B. Portions of these data were presented at the fall 1993 meeting of the Acoustical Society of America in Denver. We thank Kelly Mosteller, Chris Starkey, and Rafael Gomez for participating as human subjects and testing monkeys. Correspondence should be addressed to J. M. Sinnott, Comparative Hearing Laboratory, Department of Psychology, University of South Alabama, Mobile, AL 36688 (e-mail: jsinnott@jaguarl .usouthal.edu). mans and monkeys responded to target vowels in a repeating train of background vowels, and RTs were measured to the targets. Results showed that spectrally similar vowel pairs, even when they were discriminated perfectly as indicated by a percent correct measure, had longer RTs than spectrally dissimilar pairs. Human data provided a good match to the traditional human data based on confusion matrices or similarity measures, and monkey data were qualitatively similar to those of humans.

Recent work using MDS in conjunction with RT procedures has revealed subtle differences between humans and animals, as well as among various animal species, in perception of a variety of acoustic stimuli ranging from simple tones to complex animal sounds (C. H. Brown, Sinnott, \& Kressley, 1994; S. D. Brown, Dooling, \& O'Grady, 1988; Dooling, Brown, Park, Soli, \& Okanoya, 1987; Dooling, Park, Brown, Okanoya, \& Soli, 1987).

At present, the extent to which low-level sensory versus higher level attentional mechanisms contribute to shaping these maps remains unclear. However, in the case of human vowels, which are carriers of the speech wave, it is not unreasonable to assume that human perception may involve some mediating species-specific mechanisms, whereas monkey vowel perception would likely involve only general auditory mechanisms. For example, Kuhl (1991) has shown that human adults and infants show a "perceptual magnet" effect for the prototypes of vowel categories, whereas monkeys do not. This phenomenon shows up in a simple discrimination experiment as better generalization around good exemplars of vowels and implies that humans have stronger vowel prototypes than do monkeys.

The purpose of the present study was to further explore comparisons between human and monkey vowel 
Table 1

Formant Frequencies $(F)$ and Relative Amplitudes (A) of the Vowel Stimuli

\begin{tabular}{|c|c|c|c|c|c|c|c|c|c|c|}
\hline & $\begin{array}{c}/ \mathrm{u} / \\
(b o o)\end{array}$ & $\begin{array}{c}/ \mathbf{U} / \\
(p u t)\end{array}$ & $\begin{array}{c}/ \mathfrak{J} / \\
(p a w)\end{array}$ & $\begin{array}{c}/ \mathrm{a} / \\
(p o t)\end{array}$ & $\begin{array}{c}/ \wedge / \\
(b u t)\end{array}$ & $\begin{array}{c}/ 3 / \\
\text { (bird) } \\
\end{array}$ & $\begin{array}{c}/ \mathfrak{a} / \\
(b a t)\end{array}$ & $\begin{array}{c}/ \varepsilon / \\
(b e t)\end{array}$ & $\begin{array}{c}/ \mathrm{I} / \\
\text { (bit) }\end{array}$ & $\begin{array}{c}/ \mathrm{i} / \\
\text { (bee) }\end{array}$ \\
\hline$F 1$ & 300 & & 5 & 730 & 640 & 490 & 660 & 530 & 390 & 270 \\
\hline$F 2$ & 870 & 1020 & 840 & 1090 & 1190 & 1350 & 1720 & 1840 & 1990 & 2290 \\
\hline$F 3$ & 2240 & 2240 & 2410 & 2440 & 2390 & 1690 & 2410 & 2480 & 2550 & 3000 \\
\hline Al & -4 & -5 & -6 & -5 & -4 & -6 & -4 & -8 & -5 & -6 \\
\hline 2 & -16 & -15 & -8 & -9 & -12 & -12 & -11 & -12 & -15 & -12 \\
\hline 3 & -40 & -32 & -35 & -28 & -25 & -13 & -13 & -12 & -14 & -6 \\
\hline
\end{tabular}

processing by using the RT procedure of Sinnott (1989) and submitting the results to an MDS analysis. In addition, two different monkey species were compared with the humans: The Asian Japanese macaque (Macaca fuscata) and the African Sykes monkey (Cercopithecus albogularis). Japanese macaques are the most widely used of any monkey species in comparative speech perception research (Sinnott, 1994). In contrast, the Sykes monkey has only recently been used in speech research (C. H. Brown \& Sinnott, 1990). In addition, the Sykes monkey may have a specific adaptation for the production and/or perception of low-frequency sounds. These monkeys produce low-frequency boom spacing calls, which are very tonal sounds with dominant energy below $500 \mathrm{~Hz}$ and sound very much like the human vowel $/ \mathrm{u} /(\mathrm{C} . \mathrm{H}$. Brown \& Sinnott, 1990).

So far, comparisons of absolute vowel thresholds have not revealed any differences between humans, macaques, and Sykes monkeys for vowels with low-frequency formants (Sinnott, 1995). However, if the Sykes monkeys' specialization for low-frequency sound production extends to other aspects of perception, they might be more sensitive than other monkey species in discriminating the lower formant frequencies in human speech. For example, this ability might show up in MDS analyses as a higher weighting for the first formant $(F 1)$, compared with the macaques. Further comparisons with humans would reveal which species, if any, offered the better model of human vowel space.

\section{METHOD}

\section{Subjects}

Human subjects were 3 monolingual native speakers of American English (J.M.S., female, and C.L.S. and K.W.M., males) and 3 bilingual speakers: English-German (R.A.K., female), SpanishEnglish (R.A.F., male), and Urdu-English (W.A.S., male). The latter 2 subjects spoke English with accents. All human subjects were in their 20s except for J.M.S. (age 45) and R.A.F. (age 35). Monkey subjects were 4 Japanese macaques (M. fuscata) and 3 Sykes monkeys ( $C$. albogularis), all young males about 7 years old. All subjects had normal hearing as indicated by verbal report or by hearing tests in our laboratory.

\section{Apparatus}

The basic apparatus has been previously described in detail (e.g., C. H. Brown \& Sinnott, 1990; C. H. Brown et al., 1994). Briefly, testing took place in the free field inside a double-walled IAC booth $(3 \times 3 \times 3)$ that was lined with sound-absorbing acoustic foam (Sonex) to reduce reflections. The stimulus system included a Compac computer that stored the digitized stimuli (see below), a 12-bit D/A converter (Data Translation), programmable attenuators (Data Translation), a low-pass filter (Frequency Devices; $24 \mathrm{~dB}$ per octave) set at $4.8 \mathrm{kHz}$, an amplifier (NAD $3240 \mathrm{PE}$ ), and a loudspeaker (Polk 10) placed $84 \mathrm{~cm}$ from the subjects' heads. This system resulted in a relatively flat $( \pm 4-\mathrm{dB})$ frequency response from $125 \mathrm{~Hz}$ through $4.8 \mathrm{kHz}$. Subjects responded on a custommade, contact-sensitive metal disk mounted above a cuelight. Monkeys sat in primate chairs, and an automatic feeder delivered 190mg banana pellets (Noyes) as rewards for correct responses during the experiment.

\section{Stimuli}

The stimuli were the 10 English vowels $/ \mathrm{i} /, / \mathrm{i} /, / \varepsilon /, / \mathfrak{x} /, / \mathbf{z} /, / \mu /$ $/ \mathrm{a} /, / \mathrm{s} /, / \mathrm{u} /$, and $/ \mathrm{u} /$, modeled after the averaged adult male measurements of Peterson and Barney (1952). Vowels were synthesized using the cascade branch of the Canadian Speech Research Environment synthesizer (University of Western Ontario). They were steady-state, $300 \mathrm{msec}$ long with 20 -msec rise/fall times, with a fundamental frequency $(F 0)$ that began at $120 \mathrm{~Hz}$ and decreased linearly to $80 \mathrm{~Hz}$ at the end of the vowel. Table 1 shows the first three formant frequencies $(F 1-F 3)$ and amplitudes (A1-A3) measured relative to the overall vowel levels. The amplitudes of the formants were measured inside the test booth as follows: Vowels were played at a level of $80 \mathrm{~dB}$ (A scale). A variable filter set to the bandpass used in synthesis (see below) was centered around each formant. Inside the booth, a B\&K SPL meter and $1 / 4$-in. condenser microphone measured the level of each vowel's $F 1, F 2$, and $F 3$. The higher formant frequencies $(F 4-F 6)$ were fixed at 3300,3750 , and $4900 \mathrm{~Hz}$. Bandwidths for $F 1-F 5$ were set at the default values of $50,70,110,250$, and $1000 \mathrm{~Hz}$.

Historically, the English vowels have been classified according to several phonetic features that map onto their formants. First, vowels are classified according to advancement, which relates to the $F 2$, which in turn reflects changes in the length of the front cavity. The front vowels $(/ \mathrm{i} /, / \mathrm{I} /, / \mathcal{E} /$, and $/ \mathfrak{x} /)$ are produced with the bulk of the tongue positioned in the front of the mouth. The central vowels $(/ 3 /, / \% /$, and $/ \mathrm{a} /)$ are produced with the tongue in a more central position. The back vowels $(/ \mathrm{o} /, / \mathrm{U} /$, and $/ \mathrm{u} /)$ are produced with the tongue in the back of the mouth. $F 2$ reflects this tongue position, decreasing as the tongue moves from front to back. In addition, vowels are classified according to tongue height, or size of the constriction, which is reflected in the $F 1$. The high vowels $(/ \mathrm{i} /, / \mathrm{I} /, / \mathrm{U} /$, and $/ \mathrm{u} /)$ are made with narrow constrictions, causing a low $F 1$. The low vowels $(/ \mathfrak{x} /, / \wedge /$, and $/ \mathrm{a} /)$ are made with more open constrictions, causing a higher $F 1$. The rhotic vowel $/ 3 /$ is unique in having a very low $F 3$ and is produced by "bunching" the tongue so that two constrictions, one palatal and one pharyngeal, are produced in the supraglottal vocal tract (Delattre \& Freeman, 1967).

Figure 1 (left panel) plots the formants of the vowels relative to one another. Figure la plots vowel $F 2$ (representing advancement) versus $F 1$ (height). Note that the frequency axes are reversed in 


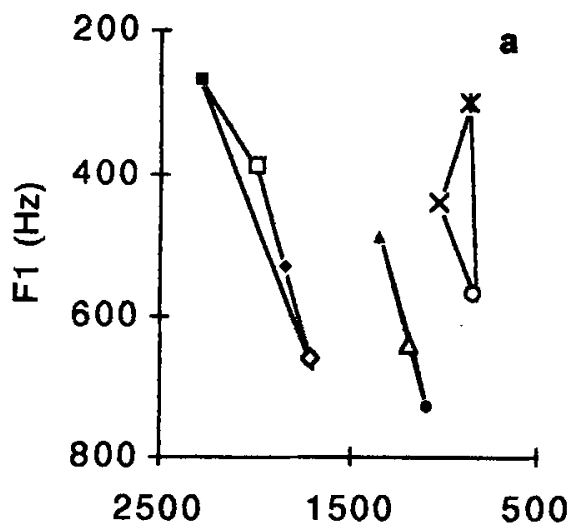

$\mathrm{F} 2(\mathrm{~Hz})$

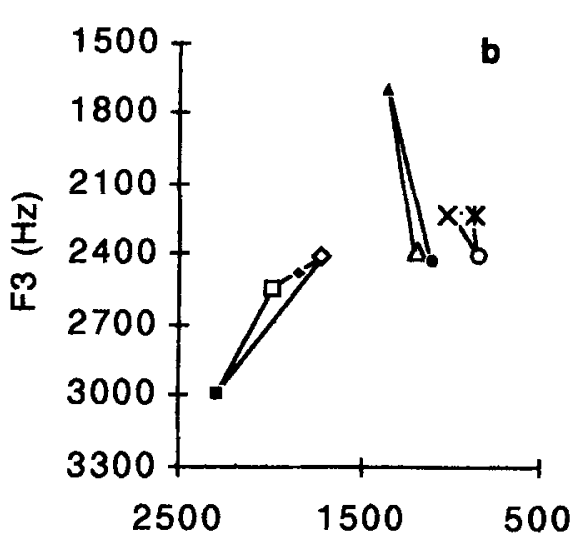

F2 $(\mathrm{Hz})$

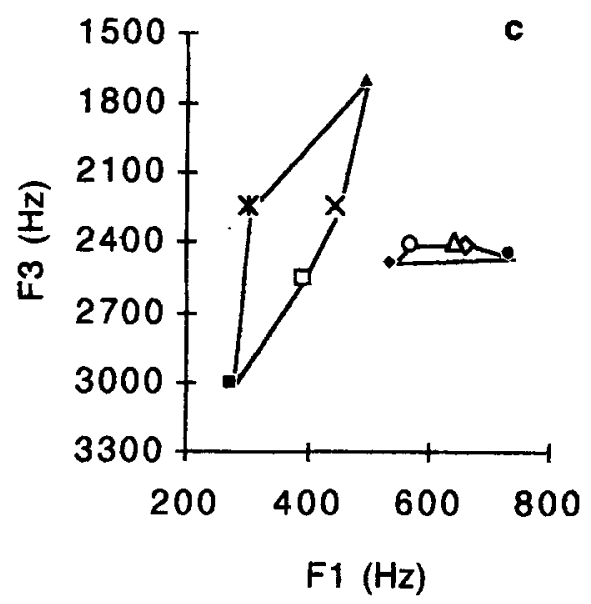

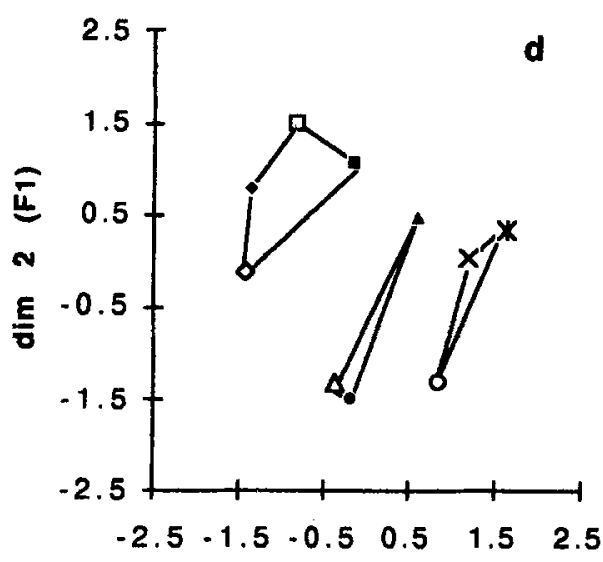

dim 1 (F2)

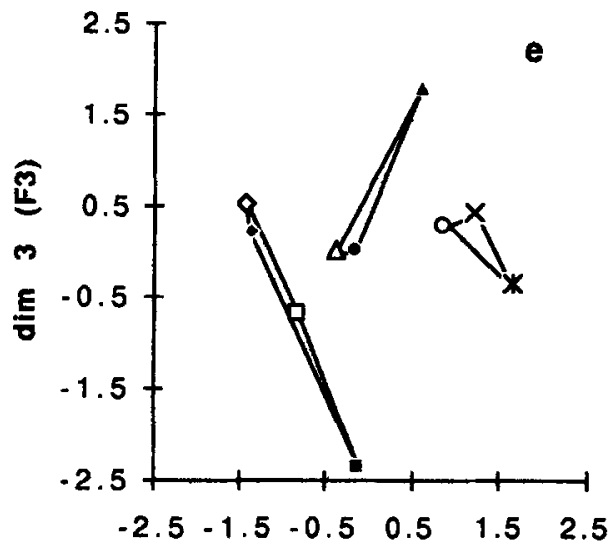

$\operatorname{dim} 1$ (F2)

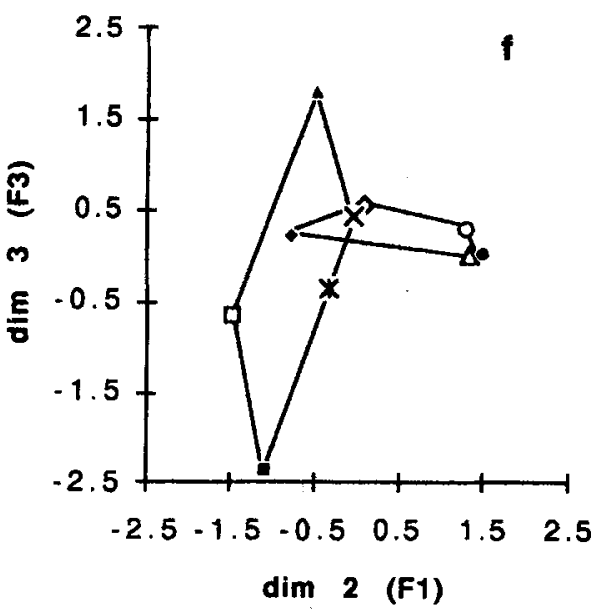

Figure 1. Left panels: Plots of English vowel formants in hertz relative to one another. Panel a: $F 2$ versus $F 1$, showing vowels grouped by $F 2$ (advancement). Panel b: $F 2$ versus $F 3$, showing vowels grouped by $F 2$. Panel c: $F 1$ versus $F 3$, showing vowels grouped by $F 1$ (height). Right panels: Perceptual maps for all subjects pooled based on 6 humans and 7 monkeys using INDSCAL. Panel d: Dimension (DIM) 1, emerging as F2 (advancement) versus DIM 2, emerging as $F 1$ (height), showing vowels grouped by $F 2$. Panel e: DIM $1(F 2)$ versus DIM 3, emerging as $F 3$ (rhoticization), showing vowels grouped by $F 2$. Panel f: DIM $2(F 1)$ versus DIM $3(F 3)$, showing vowels grouped by $F 1$ (height). 

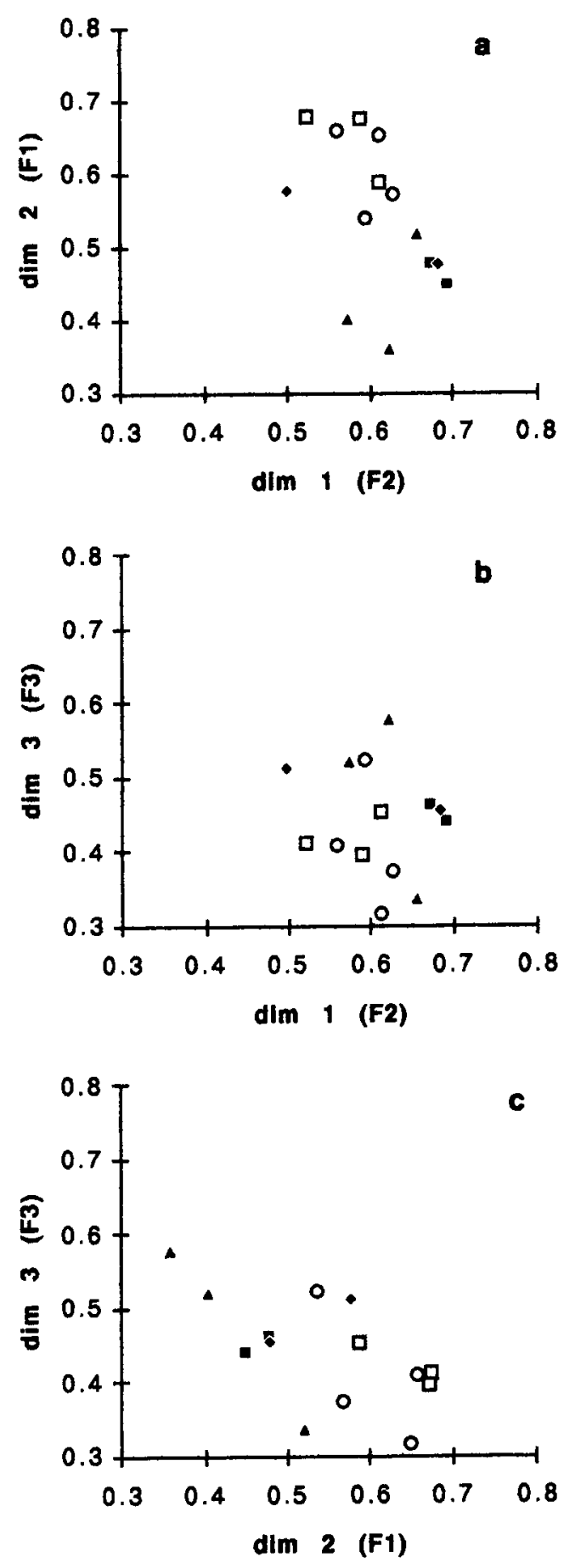

Figure 2. Perceptual weights from 6 humans and 7 monkeys using INDSCAL. Human subjects are denoted by filled symbols, monkeys by open symbols. Filled squares represent the two replications of Subject J.M.S. Filled diamonds represent two other monolingual native speakers of English. Filled triangles represent the bilingual speakers. Open circles represent the Japanese macaques; open squares represent the Sykes monkeys. Panel a: weights for Dimension (DIM) $1(F 2)$ versus DIM $2(F 1)$. Panel b: weights for DIM $1(F 2)$ versus DIM $3(F 3)$. Panel c: weights for DIM $2(F 1)$ versus DIM $3(F 3)$. order to correspond with the phonetic dimensions as traditionally portrayed in the "vowel quadrilateral" (see, e.g., Rakerd, 1984; Rakerd \& Verbrugge, 1985): The high front vowels are in the upper left, the low central vowels are at the bottom center, and the high back vowels are in the upper right. Figure $1 \mathrm{~b}$ plots vowel $F 2$ versus $F 3$ (rhoticization). Note that the vowels $/ 3 /$ and $/ \mathrm{i} /$ are at opposite ends of Dimension (DIM) 3. In Figures $1 \mathrm{a}$ and $1 \mathrm{~b}$, the vowels are grouped according to advancement (front, central, back). Figure 1c plots vowel $F 1$ versus $F 3$, and here the vowels are grouped according to height: high vowels $(F 1<500 \mathrm{~Hz})$ versus low vowels $(F \mathrm{l}>500 \mathrm{~Hz})$. For present purposes, we have split the vowel height continuum into these two groups.

\section{Procedure}

All subjects were tested with a minimal-uncertainty repeatingbackground same-different $\mathrm{AX}$ discrimination procedure that has been extensively used over the last 20 years in studies of animal psychoacoustics and speech discrimination (Sinnott, 1994, 1995). A trial began when the subject contacted the response disc during the flashing cuelight. At this time, the cuelight steadied and a background vowel began repeating at a rate of 1 per $800 \mathrm{msec}$, at a level of $60 \mathrm{~dB}$ SPL (A). After a variable time period of 1,600 $6,400 \mathrm{msec}, 2$ identical target vowels occurred representing 1 of the other 9 vowels. A subject had a 1,600-msec response interval, measured from the onset of the target, to release the disc in order to score a hit, which was immediately rewarded with a food pellet (monkeys) or the feeder click (humans). Following rewards, an intertrial interval (ITI) occurred, during which the cuelight was extinguished and trials could not be initiated.

If the subject did not respond to the target during the response interval (miss), a 3-sec TO (timeout) occurred as feedback to the subject. Control trials occurred on $20 \%$ of the trials. Here, a target occurred that was identical to the background. Responses to control trials (false alarms) and other premature releases were punished with 8-sec TOs. Control trials that were not responded to (correct rejections) were immediately followed by a suprathreshold target vowel that contained a highly salient $6-\mathrm{dB}$ intensity cue, to which all subjects could easily respond.

During each session, one vowel was chosen as the background and the nine other vowels were randomly selected as targets on test trials according to the method of constant stimuli. The total percentage of hits and false alarms and the median RT for each target vowel were recorded. Humans worked in 10- to $15-\mathrm{min}$ daily sessions that terminated after 70 rewards, and monkeys worked in 30- to 35-min sessions that terminated after 140 rewards. Subjects were tested with a different vowel each day, and the order of testing the 10 vowel backgrounds was randomized among subjects. Humans were tested for a total of six sessions for each background, and monkeys were tested for eight, which allowed all subjects to reach asymptotic levels of performance. The last four sessions were used for data analysis.

An RT matrix was constructed from data pooled across the four sessions for each background. RTs were first converted to logs to accommodate skewed RT distributions and to equate proportional increases in RT for fast and slow subjects. RTs were entered into a $10 \times 10$ matrix representing all pairwise comparisons, with background vowels in columns and target vowels in rows. The matrix was then folded by averaging the corresponding cells in the upper and lower halves and excluding the diagonal cells.

The resulting half-matrix was converted to a spatial map in three dimensions using the ALSCAL program (Statistical Package for the Social Sciences). Data were analyzed using the ordinal/ similarity option, which treats the data as rank ordered and treats higher RTs as reflecting greater similarity. (An additional analysis using the interval option revealed no differences in the two sets of data.) We first used the INDSCAL model, which produces one 

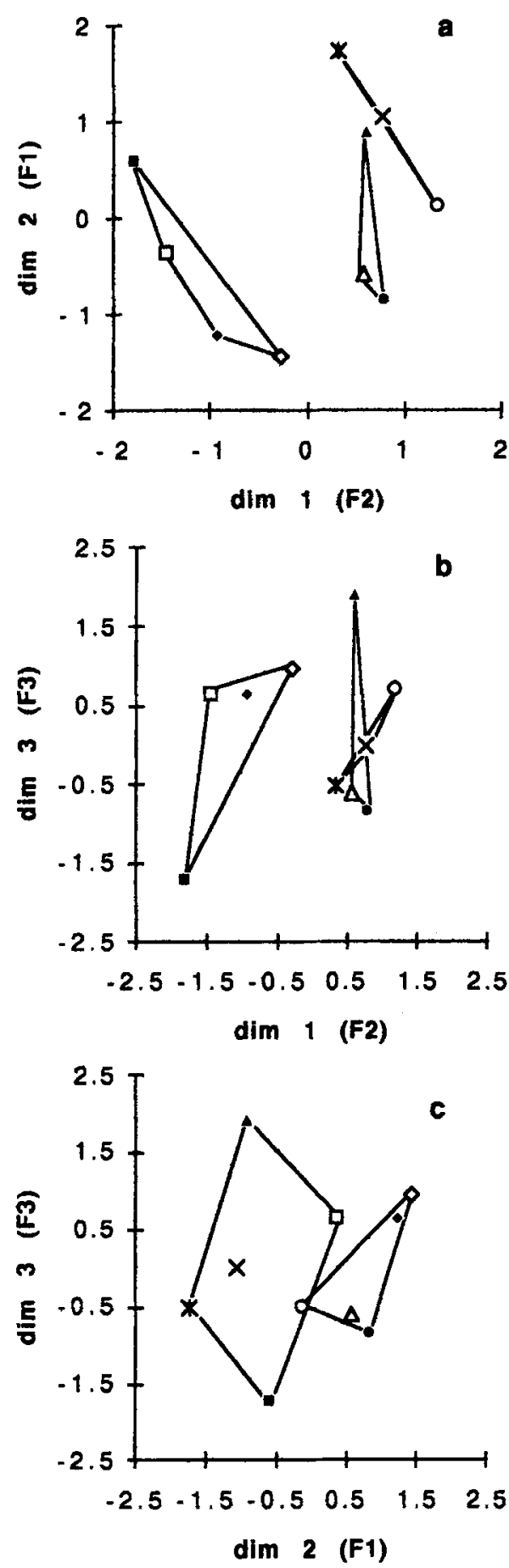
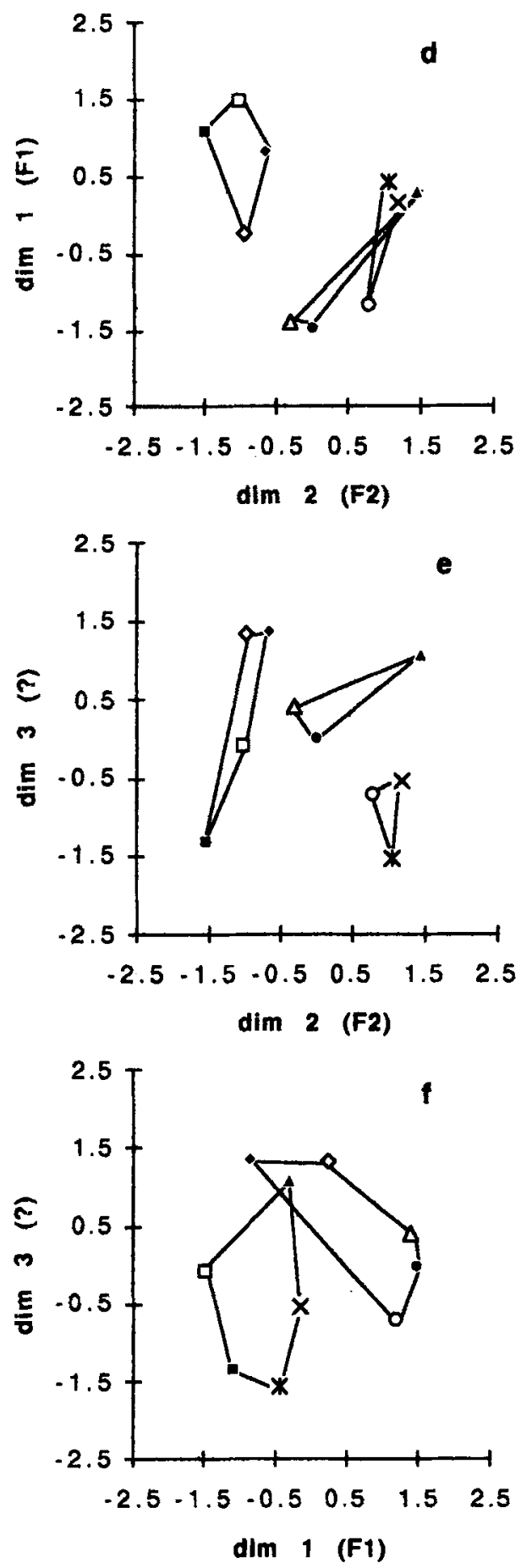

Figure 3. Left panels: Human perceptual maps based on 6 humans, including two replications from Subject J.M.S. Panel a: Dimension (DIM) 1, emerging as $F 2$ (advancement) versus DIM 2, emerging as $F 1$ (height), showing vowels grouped by F2. Panel b: DIM $1(F 2)$ versus DIM 3, emerging as F3 (rhoticization), showing vowels grouped by $F 2$. Panel c: DIM $2(F 1)$ versus DIM $3(F 3)$, showing vowels grouped by $F 1$. Right panels: Monkey perceptual maps based on 4 Japanese macaques and 3 Sykes monkeys. Panel d: DIM 2, emerging as $F 2$ (advancement) versus DIM 1, emerging as $F 1$ (height), and showing vowels grouped by $F 2$. Panel e: DIM 2 (F2) versus DIM 3 (?), which could not be identified, and showing vowels grouped by $F 2$. Panel f: DIM $1(F 1)$ versus DIM 3 (?), showing vowels grouped by $F 1$. 

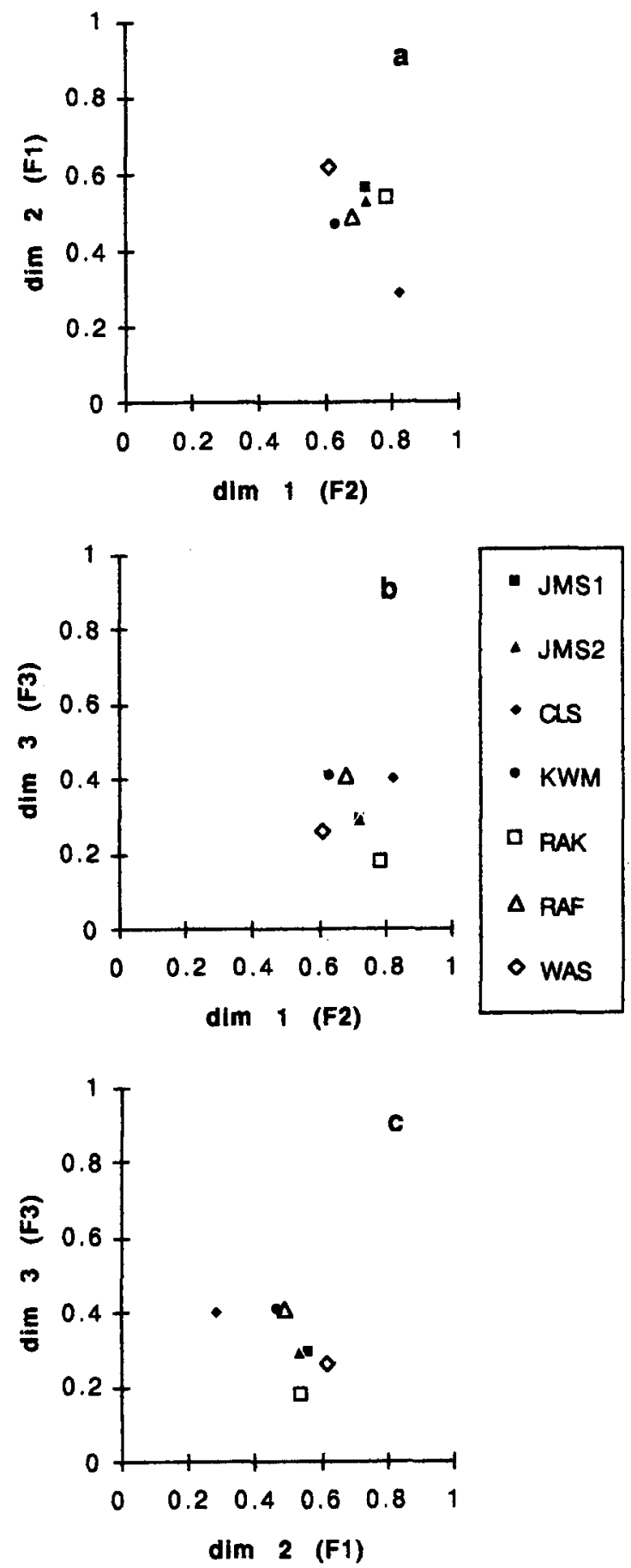

Figure 4. Human perceptual weights based on 6 humans, including two replications from Subject J.M.S. Monolingual English speakers (J.M.S., C.L.S., and K.W.M.) are denoted by filled symbols, bilingual speakers (R.A.K., R.A.F., and W.A.S.) by open symbols. Panel a: weights for Dimension (DIM) $1(F 2)$ versus DIM $2(F 1)$. Panel b: weights for DIM $1(F 2)$ versus DIM $3(F 3)$. Panel c: weights for DIM $2(F 1)$ versus DIM $3(F 3)$.

matrix for all the submitted data and shows individual subject weights and weirdness scores for each dimension. We then analyzed all subjects using the simple Euclidean model to obtain individual maps for each subject.
Human subject J.M.S. ran through the whole testing sequence twice, once at the beginning of the study and once at the end, approximately 6 months apart. The purpose of this was to examine within-subject variability in the MDS analysis.

\section{RESULTS}

Human subjects responded to all target vowels with $100 \%$ accuracy, and monkeys responded with greater than $90 \%$ accuracy. False alarm rates were less than $5 \%$ for all subjects in all sessions. RTs showed overlap for humans and monkeys: Mean RTs for the human subjects averaged over all sessions ranged from 263 to $337 \mathrm{msec}$. Mean RTs for the macaques ranged from 293 to $405 \mathrm{msec}$, and for the Sykes, 328 to $348 \mathrm{msec}$.

\section{Humans and Monkeys Analyzed Together, Using INDSCAL}

In this analysis, the 6 humans (including two replications from Subject J.M.S.) and the 7 monkeys were analyzed together using INDSCAL. The perceptual maps for all subjects pooled are presented in Figure 1 (right panel).

Despite the pooling of human and monkey data, common dimensions emerged. Figure $1 \mathrm{~d}$ shows the map for DIM 1 versus DIM 2, which can be compared with the corresponding formant plot in Figure 1a. DIM 1 emerged as $F 2$ (advancement), separating the front, central, and back vowels from left to right. DIM 2 emerged as $F 1$ (height), separating the high and low vowels from top to bottom. There were several ways in which the perceptual map differed from the vowel plot: Along DIM $1(F 2)$, the front vowels $(/ \mathrm{i} /, / \mathrm{I} /, / \varepsilon /$, and $/ \mathfrak{a} /)$ were out of order; the rhotic vowel $/ 3 /$ was shifted toward the back vowels, and the back vowel $/ 0 /$ was shifted toward the central vowels. Along DIM $2(F 1)$, the front vowel space was shrunk, largely because /i/ was out of order, perceived with a higher $F 1$ than was $/ \mathrm{I} /$.

Figure 1e shows the map for DIM 1 versus DIM 3. DIM 3 emerged as $F 3$ (rhoticization), separating out the vowel $/ 3 /$ on top, with its low $F 3$, from the other vowels, in particular from /i/ on the bottom, with its high $F 3$. Comparing this map with the corresponding formant plot in Figure $1 \mathrm{~b}$, we see that the match was quite good in separating out the vowels $/ 3 /$ and $/ \mathrm{i} /$, both with extreme $F 3 \mathrm{~s}$, in both plots.

Figure $1 \mathrm{f}$ shows the map for DIM 2 versus DIM 3. This map can be compared with the corresponding formant plot in Figure 1c. As in the formant plot, vowels are grouped here according to $F 1$ (height), and vowels with $F 1 \mathrm{~s}$ below and above $500 \mathrm{~Hz}$ are separated into two groups. There is a good match except that the vowels / $/$ / and $/ \mathrm{u} /$ are reversed along DIM $2(F 1)$, and the vowels $/ \varepsilon /$ and $/ \mathfrak{x} /$ are shifted toward the higher vowels.

The pooled map does not allow us to determine whether the mismatches observed were due to aspects of human or monkey perception, or both. Nevertheless, the pooled analysis is necessary to derive the weights for the different dimensions for humans versus monkeys. 

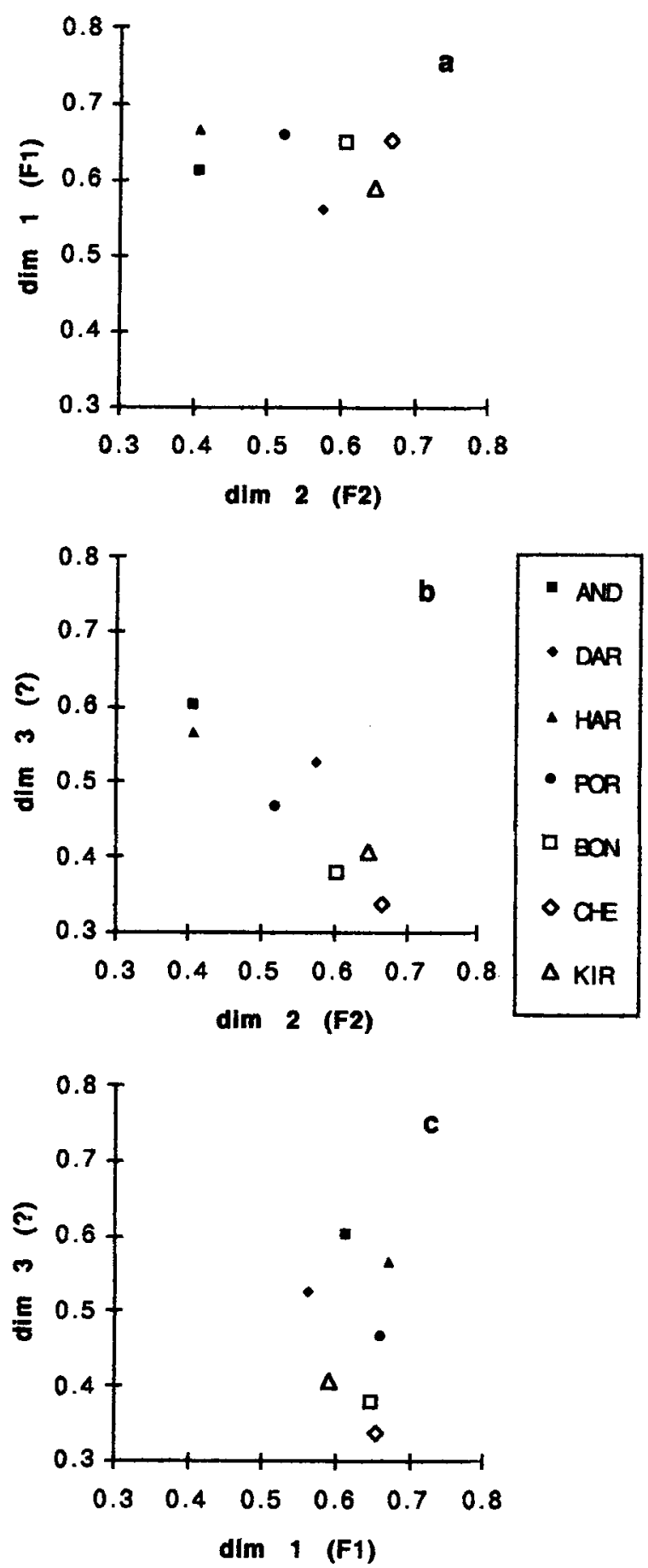

Figure 5. Plots of perceptual weights for 4 Japanese macaques (AND, DAR, HAR, and POR, filled symbols) and 3 Sykes monkeys (BON, CHE, and KIR, open symbols). Panel a: weights for Dimension (DIM) $2(F 2)$ versus DIM $1(F 1)$. Panel b: weights for DIM 2 (F2) versus DIM 3 (?). Panel c: weights for DIM 1 (F1) versus DIM 3 (?).

Figure 2 shows plots of human versus monkey weights. Figure 2a shows the plot of DIM $1(F 2)$ versus DIM 2 $(F 1)$, which separated most of the human weights (filled symbols) from the monkey weights (open symbols). All the humans except for 1 weighted DIM 1 over DIM 2, whereas the monkeys weighted these dimensions equally. Figure $2 \mathrm{~b}$ shows the plot of DIM $1(F 2)$ versus DIM 3 $(F 3)$. Here there was much overlap between humans and monkeys, and both groups weighted DIM 1 over DIM 3. Figure 2c shows the plot of DIM $2(F 1)$ versus DIM 3 $(F 3)$. Humans weighted these dimensions equally, but the monkeys weighted DIM $2(F 1)$ more heavily than did the humans. The weight plots can be summarized as follows: For humans, DIM $1(F 2)>$ DIM $2(F 1)=$ DIM $3(F 3)$. For monkeys, DIM $1(F 2)=\operatorname{DIM} 2(F 1)>\operatorname{DIM} 3(F 3)$.

A two-way analysis of variance (ANOVA) was performed to examine the interaction of species (human vs. monkey) and dimension $(1,2,3)$ on weights. Only the first run of Subject J.M.S. was included in this analysis. Results indicated a significant interaction $[F(2,22)=$ $13.51, p<.01$ ], verifying the effects seen in Figure 2 . In addition, when each of the monkey species was compared separately with humans, both interactions of species $\times$ dimension were significant [humans vs. macaques, $F(2,16)=9.45, p<.01$; humans vs. Sykes, $F(2,14)=$ $15.18, p<.01]$. None of the plots showed any differences between between the monolingual and bilingual speakers of English or between the two monkey species. The two replications of human Subject J.M.S. showed similar weightings.

Weirdness scores indicate a subject's deviation from the average weight for a particular dimension. A subject with a weight proportional to the average weight has a weirdness score of 0.0 , and a subject with one large weight and many small weights has a weirdness score near 1.0, the maximum value. Weirdness scores for humans ranged from .1015 to .2726 , and for monkeys ranged from .0312 to .2117 . An analysis of weirdness scores showed that they did not differentiate humans from monkeys $[F(1,11)=1.70, p>.10]$.

\section{Human Analysis Using INDSCAL}

In order to determine more precisely the form of the human perceptual map and to examine possible differences between the monolingual and bilingual speakers of English, the seven human matrices were analyzed together in this INDSCAL analysis, including the two replications from Subject J.M.S. The results are shown in Figure 3 (left panel).

Figure 3a shows the human perceptual map for DIM 1 versus DIM 2, with DIM 1 emerging as $F 2$ and DIM 2 emerging as $F 1$. In general, the human map was a good match to the corresponding formant plot in Figure la. The order along both DIM $1(F 2)$ and DIM $2(F 1)$ in the front vowels followed that in the formant plot. However, along DIM 1 , the rhotic vowel $/ 3$ / was shifted slightly toward the back vowels, and back vowel/ $\mathrm{u} /$ was shifted more centrally.

Figures $3 b$ and $3 c$ show DIM 1 versus DIM 3 and DIM 2 versus DIM 3 , respectively. For both maps, DIM 3 emerged clearly as $F 3$. In Figure $3 \mathrm{~b}$, there was overlap between the central and back vowels, which did not occur in the formant plot (cf. Figure 1b). In Figure 3c, the vowel / $/$ / overlapped into the high vowel group, which did not occur in the formant plot in Figure lc. 

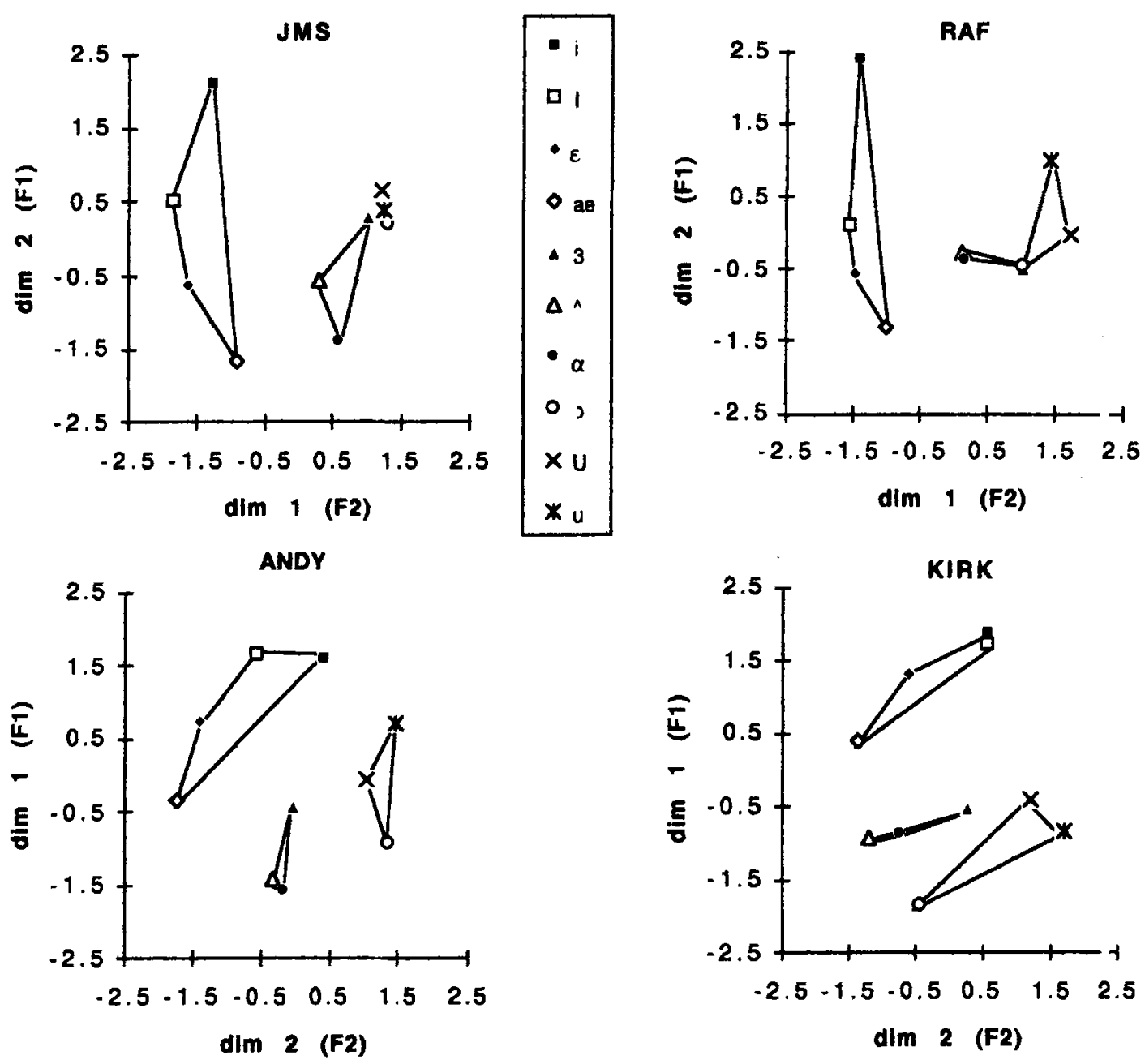

Figure 6. Plots of 4 individual subjects showing DIM 1 and DIM 2.

Figures $4 a, 4 b$, and $4 c$ show the human subjects' weights analysis. These plots did not show any differentiation between the monolingual and bilingual speakers of English for any dimensions, as verified by a two-way ANOVA $[F(2,8)=2.27, p>.10]$. Further, an analysis of weirdness scores did not differentiate the monolingual and bilingual English speakers $[F(1,4)=.12, p>.25]$.

\section{Monkey Analyses Using INDSCAL}

In order to determine more precisely the form of the monkey perceptual map and to examine possible differences between the Japanese macaques and the Sykes monkeys, the 7 monkeys were analyzed together using INDSCAL. Figure 3 (right panel) shows the resulting perceptual maps, which can be compared with the human maps in Figure 3 (left panel).

Figure 3d shows the monkey map of DIM 2 versus DIM 1. Here, in contrast to the human map, DIM 1 emerged as $F 1$, and DIM 2 emerged as $F 2$. Therefore, in order to orient the monkey map with that for humans, the monkey map was rotated once clockwise and then re- flected along DIM 1 (C. H. Brown et al., 1994). Vowels are grouped by $F 2$ along DIM 2 . Close inspection of Figures $3 \mathrm{a}$ and $3 \mathrm{~d}$ show that the most notable perceptual differences were as follows: Along DIM 1 , monkeys misperceived the $F 1$ of $/ \mathrm{i} /$, and along DIM 2 , the maps of the central and back vowels overlapped, due to the misperception of the $F 2$ of the vowel $/ 3$.

Figure $3 \mathrm{e}$ shows the monkey map of DIM 2 versus DIM 3. Note that, in contrast to the human map, DIM 3 could not be readily identified as $F 3$. Although $/ 3 /$ and /i/ are on opposite sides of DIM 3, they do not readily stand out from the other vowels, and the extreme displacements along DIM 3 were received for the vowels $/ \mathbf{u} /, / \mathfrak{a} /$, and $/ \varepsilon /$. Thus DIM 3 could not be readily identified for monkeys. Figure $3 \mathrm{f}$ shows the plot of DIM 1 versus DIM 3. For monkeys, unlike humans, the vowels $/ \varepsilon /$ and $/ æ /$ are shifted toward the high vowels. Again, DIM 3 could not be readily identified as $F 3$.

Figure 5 shows the monkey weight analyses, which revealed some differences between the two monkey species. Figure 5a shows the plot of DIM $2(F 2)$ versus DIM $1(F 1)$. 
For macaques, DIM 1 was weighted over DIM 2, whereas for Sykes monkeys, these two dimensions were weighted equally. Figure 5 b shows the plot of DIM $2(F 2)$ versus DIM 3 (?). Here, macaques weighted these dimensions equally, but the Sykes monkeys weighted DIM 2 over DIM 3. Figure $5 \mathrm{c}$ shows the plot of DIM $1(F 1)$ versus DIM 3 (?). Here both species weighted DIM 1 over DIM 3. These results can be summarized as follows: For macaques, DIM $1(F 1)>$ DIM $2(F 2)=$ DIM 3 (?). For Sykes, DIM $1(F 1)=$ DIM $2(F 2)>$ DIM $3($ ?). An ANOVA revealed a significant interaction between species and dimensions $[F(2,10)=24.31, p<.01]$, verifying the visual observations in Figure 5. An analysis of weirdness scores revealed no differences between the two species $[F(1,5)=.37, p>.25]$.

Our original hypothesis was that the Sykes monkeys might weigh $F 1$ relatively more heavily than would the macaques because of their specialization for lowfrequency sound production. Our results did not verify this hypothesis, because in fact the Japanese macaques weighted $F 1$ relatively more heavily than did the Sykes monkeys.

\section{Individual Analyses Using the Euclidean Model}

The INDSCAL model provides a pooled perceptual map and a comparison of subject weights for the different emergent dimensions, but it does not provide any individual maps. In order to compare the form of the vowel space for individual humans and monkeys, all subjects were analyzed using the simple Euclidean model. Examples of some individual perceptual maps for DIM 1 versus DIM 2, and grouping of the vowels by $F 2$, are shown in Figure 6.

The data for 2 human subjects are shown: J.M.S., a monolingual English speaker, and R.A.F., the SpanishEnglish bilingual speaker. Except for some minor quantitative differences in the areas of the front, central, and back vowel spaces, the maps are for the most part similar. The data for 2 monkey subjects are also shown: Andy, a Japanese macaque, and Kirk, a Sykes monkey. As in Figure 5 , the maps were oriented to best match the human maps, with $F 2$ along the $x$-axis. The monkey "front," "central," and "back" vowel spaces appear similar to those in the human plots, although they are rotated slightly clockwise. Further examination of the other subjects' maps did not reveal any consistent differences between the human and monkey vowel spaces for DIM 1 and DIM 2.

\section{DISCUSSION}

\section{Comparison With Other \\ Human MDS Vowel Analyses}

The present analyses show that vowel $F 2, F 1$, and $F 3$, representing the classic English vowel features of advancement, height, and rhoticization, emerged in the human data. The present RT measure provides a good match to other human data based on confusion matrices or similarity ratings.
Shepard (1972) used a three-dimensional analysis of the confusion matrices of the Peterson-Barney (1952) vowels spoken in the $/ \mathrm{hVd} /$ context. He found the traditional English vowel classification according to $F 2, F 1$, and $F 3$. Rotating his axes so that each would best agree with the average center frequency of the vowel formants, he found that his dimensions were correlated with the formants as follows: .98 for $F 2, .88$ for $F 1$, and .82 for $F 3$, thus indicating the salience of $F 2$.

Pols et al. (1969) used the Shepard (1972) model to analyze a martix of 11 Dutch vowels derived from a rating procedure. The two primary dimensions of $F 2$ and $F 1$ emerged. Since Dutch contains no $/ 3 /$, this vowel was not used and thus $F 3$ did not emerge as DIM 3, and the authors could not readily identify their third dimension. One native speaker of Japanese was also tested and performed similarly to the Dutch speakers. The authors concluded that $F 2$ and $F 1$ are the most important factors in vowel perception.

Singh and Woods (1970) used Shepard's (1972) procedure to analyze dissimilarity ratings of English vowels spoken in isolation. They interpreted the resulting dimensions in terms of phonetic features. Three dimensions emerged as follows: advancement $(F 2)$ was the strongest, with $70 \%$ of variance accounted for, compared with $15 \%$ for height $(F 1)$ and $15 \%$ for retroflexion (rhoticization) $(F 3)$. The authors also found that when the vowel $/ 3 /$ was eliminated from the data, only the first two dimensions were necessary to classify the vowels. These data are similar to those in the present study, in which the advancement feature $(F 2)$ was dominant for humans.

Rakerd (1984) used an individual differences model and a rating procedure to compare maps for vowels spoken steady state in a CVC context and classified in terms of phonetic features. DIM 1 was advancement $(F 2)$, and DIM 2 was height $(F 1)$. Since this study did not include /3/, DIM 3 did not emerge as rhoticization $(F 3)$, but as tenseness, separating the short and long vowels. The synthesized vowels in the present study did not include duration differences, so we cannot make comparisons here with Rakerd. Nevertheless, the results for DIM 1 and DIM 2 are clearly similar to ours and clearly indicate the dominance of $F 2$ for human subjects.

Fox et al. (1995) recently used MDS to compare the perception of English and Spanish vowels by native English and Spanish listeners. They used seven naturally spoken English vowels $(/ \mathbf{i} /, / \mathbf{l} /, / \mathbf{\varepsilon} /, / \mathfrak{\gtrless} /, / \wedge /$, and $/ \mathbf{a} /)$ and three Spanish vowels $(/ \mathrm{i} /, / \mathrm{e} /$, and $/ \mathrm{a} /)$. All vowels were rated together on a similarity/dissimilarity scale. For the English listeners, three dimensions emerged, but for the Spanish, only two dimensions emerged. Both groups perceived DIM 1 as height $(F 1)$. The English listeners perceived DIM 2 as advancement, but for the Spanish, DIM 2 was uninterpretable. These results differ from those of all the other human studies of vowel perception in that $F 2$ did not emerge as the dominant dimension; however, as the authors admitted, this was probably due to the nature of their stimulus set, which did not include any back vowels such as $/ \mathrm{u} /, / \mathbf{u} /, / \mathrm{o} /$, or $/ \mathrm{o} /$. 
The present study also included some nonnative speakers of English in order to determine whether they might have a different weighting of the emergent dimensions or possibly have differently shaped vowel spaces from those of native English listeners. For example, Spanish has only five vowels $(/ \mathrm{i} /, / \mathrm{e} /, / \mathrm{a} /, / \mathrm{o} /, / \mathrm{u} /)$ and does not differentiate the spectrally similar English vowels $/ \mathrm{i} /-/ \mathbf{I} /, / \varepsilon /-/ \mathfrak{a} /, / \wedge /-/ \mathbf{a} /$, and $/ \mathrm{u} /-/ \mathrm{u} /$. (Many readers may be familiar with how Spanish speakers confuse the English /i/ with / $/$ /: "GEEve me a lEEtle bEEt of thEEs.") However, no systematic differences showed up in the weights or the maps of the native and nonnative speakers of English. For example, Spanish speaker R.A.F. very nicely differentiated $/ \mathrm{i} /$ and $/ \mathrm{I} /$ (Figure 6).

The rhotic vowel /3/ does not occur in Spanish, Urdu, or German, languages spoken by our bilingual subjects. Yet these subjects' weights for DIM $3(F 3)$ did not differ from those of our monolingual English listeners. These data indicate that all our bilingual subjects had normal perceptions of English /3/, either from learned exposure to English or possibly from some innate human-specific auditory capacity.

\section{Humans and Monkeys Compared}

In their study of Dutch vowels, Pols et al. (1969) concluded that their subjects were simply using physical information in the stimuli, but as we discuss below with respect to monkeys, this might not be entirely the case for humans.

For the most part, the plots of our humans and monkeys were similar in that both $F 2$ and $F 1$ emerged as common dimensions. However, the weight analyses showed that $F 2$ was weighted over $F 1$ for humans, whereas $F 1$ and $F 2$ were weighted equally for the Sykes monkeys, and $F 1$ was weighted over $F 2$ for the Japanese macaques. Presumably, the monkeys may have given relatively more weight to $F 1$ because it was overall the most intense formant in any vowel $(F 2$ was anywhere from 2 to $12 \mathrm{~dB}$ lower in amplitude than was $F 1$ ). Thus the monkeys may have perceived $F 1$ as the loudest formant because even though their absolute sensitivity falls off below $1 \mathrm{kHz}$ (as does that for humans), their equal-loudness contours tend to flatten out across frequencies at high levels, so that a $250-\mathrm{Hz}$ tone and a $2-\mathrm{kHz}$ tone are equally loud at $60 \mathrm{~dB}$ SPL (Pfingst, Hienz, Kimm, \& Miller, 1975). For humans, even if $F 1$ was the loudest, $F 2$ was more salient. This result may support a phonetic hypothesis that $F 2$ is the single most important formant for humans in speech perception (Liberman, Cooper, Shankweiler, \& Studdert-Kennedy, 1967).

On the other hand, some evidence from the present study suggests that humans were sensitive to formant amplitudes. For example, note how the back vowel /u/ was shifted centrally for humans (Figure $3 a$ ). One possible explanation for this result is that humans were less sensitive to $F 2$ in this particular vowel because its $F 2$ was lower in amplitude $(-12 \mathrm{~dB})$ than was $F 1$ (Table 1 ).

Two other studies have examined vowel discrimination in animals using MDS, and both appear to show the dom- inance of $F 1$ over $F 2$ for animals. Kojima and Kiritani (1989) compared humans and chimpanzees in their perception of Japanese and French vowels. The humans weighted $F 2$ over $F 1$, as indicated by shorter RTs for discriminating vowels along the $F 2$ axis. In contrast, the chimpanzees weighted $F 1$ over $F 2$, as indicated by shorter RTs for discrimination along the $F 1$ axis. Most notably, all chimpanzees had trouble discriminating /i/ from /u/, which share a common $F 1$ but a very different $F 2$.

Dooling and Brown (1990) used MDS with parakeets to compare how these birds perceived phonetic versus talker differences. They used the vowels $/ \mathrm{i} /, / \varepsilon /, / \mathbf{a} /$, and $/ \mathrm{u} /$ with four different talkers. Results indicated that DIM 1 was most highly correlated with $F 1(.752)$ and DIM 2 was most highly correlated with $F 2(-.901)$. However, no humans were tested in this experiment for comparison.

Another difference between humans and monkeys in the present study was that the monkeys did not have a clear representation of $F 3$ in their INDSCAL perceptual maps. If monkeys are giving a true representation of the purely psychoacoustical aspects of vowel processing, then this outcome would suggest that $F 3$ itself, which was anywhere from 0 to $36 \mathrm{~dB}$ lower in amplitude than $F 1$, was not very salient, and that the weight that humans gave to $F 3$ may also reflect some "special" aspect of human phonetic coding. We leave open the question as to whether "special" reflects a learned or innate capacity, however.

To summarize, it appears that the dimensions that emerged for humans using these simple vowel stimuli may in fact have been influenced by some phonetic representation of the vowels, rather than by just vowel acoustics, as presumably was the case for the monkeys. However, if only the first two dimensions of the analyses are considered, it should be apparent that the perceptual maps of both monkey species were, for the most part, good approximations to the human maps. For example, when the individual Euclidean maps were studied in isolation, it was difficult to find any definitive characteristics that distinguished all the monkey maps from all the human maps. These results suggest that either monkey species would be a good candidate for additional studies of vowel processing in more complex contexts that would likely shed light on higher levels of phonetic processing. We leave open the possibility that more striking differences between humans and monkeys might emerge using more complex natural stimuli containing vowels in consonantal context.

\section{Comparison of Monkey Species}

Our weights analysis on the monkeys indicated that the Japanese macaques weighted $F 1$ over $F 2$, whereas the Sykes monkeys weighted $F 1$ and $F 2$ equally. Thus, our original hypothesis that the Sykes monkeys were more specialized than the macaques for low-frequency sound perception was not supported. It is important to note, however, that although the Sykes monkeys use lowfrequency formants in their "booms" (C. H. Brown \& Sinnott, 1990), there is at present no evidence that formant differences in the booms are important for the 
species. In fact, the booms of our own laboratory animals sound very much alike. Thus, the Sykes monkeys may not be particularly specialized to perceive lowfrequency formant differences in booms (or vowels).

In contrast, it is known that the "coo" system of the Japanese macaques is based on highly variable fundamental frequency $(F 0)$ patterns that convey important information about both speakers' emotional states and environmental contexts (Green, 1975). In our laboratory, it is very easy to identify our Japanese macaques by their voice variation. The $F 0$ s of these monkeys typically range between 300 and $700 \mathrm{~Hz}$ (Green, 1975), overlapping the range of the $F 1$ in human vowels. Possibly, the Japanese monkeys are predisposed to attend to this frequency region because it is so important in their own natural communication system.

\section{REFERENCES}

Brown, C. H., \& Sinnotr, J. M. (1990). The perception of complex acoustic patterns in noise by Blue monkey (Cercopithecus mitis) and human listeners. International Journal of Comparative Psychology, 4, 79-90.

Brown, C. H., Sinnott, J. M., \& Kressley, R. A. (1994). Perception of chirps by Sykes's monkeys (Cercopithecus albogularis) and humans (Homo sapiens). Journal of Comparative Psychology, 108, 243-251.

Brown, S. D., Dooling, R. J., \& O'Grady, K. E. (1988). Perceptual organization of acoustic stimuli in budgerigars (Melopsittacus undulatus): III. Contact calls. Journal of Comparative Psychology, 102, 236-247.

DelatTRe, P. J., \& Freeman, D. (1967). A dialect study of American R's by X-ray motion picture. Linguistics, 44, 29-68.

Dooling, R. J., \& BRown, S. D. (1990). Speech perception by budgerigars (Melopsittacus undulatus): Spoken vowels. Perception \& Psychophysics, 47, 568-574.

Dooling, R. J., Brown, S. D., Park, T. J., Soli, S. D., \& Okanoya, K. (1987). Perceptual organization of acoustic stimuli by budgerigars (Melopsittacus undulatus): 1. Pure tones. Journal of Comparative Psychology, 101, 139-149.

Dooling, R. J., Park, T. J., Brown, S. D., OKanoya, K., \& Solı, S. D. (1987). Perceptual organization of acoustic stimuli by budgerigars (Melopsittacus undulatus): 11. Vocal signals. Journal of Comparative Psychology, 101, 367-381.

Fox, R. A., FlegE, J. E., \& Munro, M. J. (1995). The perception of English and Spanish vowels by native English and Spanish listeners: A multidimensional scaling analysis. Journal of the Acoustical Society of America, 97, 2540-2551.
GREEN, S. (1975). Variation of vocal pattern with social situation in the Japanese monkey: A field study. In L. Rosenbloom (Ed.), Primate behavior (Vol. 4, pp. 1-101). New York: Academic Press.

KoJima, S., \& Kiritani, S. (1989). Vocal-auditory functions in the chimpanzee: Vowel perception. International Journal of Primatology, 10, 199-213.

KUHL, P. K. (1991). Human adults and human infants show a "perceptual magnet effect" for the prototypes of speech categories, monkeys do not. Perception \& Psychophysics, 50, 93-107.

Liberman, A. M., Cooper, F. S., Shankweiler, D. P., \& StuddertKENNEDY, M. (1967). Perception of the speech code. Psychological Review, 74, 431-461.

Peterson, G. E., \& Barney, H. L. (1952). Control methods used in the study of vowels. Journal of the Acoustical Society of America, 24, 175-184.

Pfingst, B. E., Hienz, R. D., Kimm, J., \& Miller, J. (1975). Reaction time procedure for measurement of hearing: I. Suprathreshold functions. Journal of the Acoustical Society of America, 57, 421-430.

Pols, L. C. W., van der Kamp, L. J., \& Plomp, R. (1969). Perceptual and physical space of vowel sounds. Journal of the Acoustical Society of America, 46, 458-467.

RAKERD, B. (1984). Vowels in consonantal context are perceived more linguistically than are isolated vowels: Evidence from an individual differences scaling study. Perception \& Psychophysics, 35, 123-136.

RAKERD, B., \& VERBRUGGE, R. R. (1985). Linguistic and acoustic correlates of the perceptual structure found in an individual differences scaling study of vowels. Journal of the Acoustical Society of America, 77, 296-301.

Schiffman, S. S., Reynolds, M. L., \& Young, F. W. (1981). An introduction to multidimensional scaling. Orlando, FL: Academic Press.

ShEPARD, R. N. (1972). Psychological representation of speech sounds. In E. Davis \& P. Denes (Eds.), Human communication: A unified view (pp. 67-113). New York: McGraw-Hill.

SingH, S., \& WoODs, D. R. (1970). Perceptual structure of 12 American English vowels. Journal of the Acoustical Society of America, 49, $1861-1866$.

SinNoTT, J. M. (1989). Detection and discrimination of synthetic English vowels by Old World monkeys (Cercopithecus, Macaca) and humans. Journal of the Acoustical Society of America, 86, 557-565.

SinNotT, J. M. (1994). Comparisons of human and monkey differential sensitivity to speech, non-speech and monkey speech sounds. In J. Menon (Ed.), Current topics in acoustic research (pp. 355-364). Trivandrum, India: Research Trends.

SinNort, J. M. (1995). Methods to assess the processing of speech sounds by animals. In G. Klump, R. Dooling, R. Fay, \& W. Stebbins (Eds.), Methods in comparative psychoacoustics (pp. 281-292). Basel: Birkhauser.

(Manuscript received June 26, 1996; revision accepted for publication December 6, 1996.) 